\title{
LETTERS
}

doi:10.1017/S1041610207005947

\section{Reduced awareness of executive dysfunction in Alzheimer's disease is associated with increased carer burden}

Previous research has shown that the presence of behavioral and psychological symptoms (BPSD) can increase the burden of care in dementia (e.g. Donaldson et al., 1998). Impaired executive functions, also called "dysexecutive syndrome" (Wilson et al., 1996), are frequent in dementia and can adversely affect social behavior in everyday life. Reduced awareness of cognitive deficits is associated with impaired executive functions, and may play a significant role in the onset and maintenance of BPSD. A recent paper by Davis and Tremont (2007) demonstrated that dysfunction of frontal systems is associated with increased burden of care in dementia, even after controlling for clinical severity and caregiver mood. In this letter, we report results that support this finding.

We studied older adults with mild Alzheimer's disease (AD) who were recruited into a longitudinal trial of physical activity. We used the Dysexecutive Questionnaire (DEX) to assess executive dysfunction. The DEX is part of the Behavioural Assessment for Dysexecutive Syndrome (Wilson et al., 1996). This questionnaire requires the respondent to rate themselves (or the patient, in the case of the informant version of the $\mathrm{DEX}$ ) on 20 problems spanning cognitive, behavioral and emotional domains. The difference between the informant and patient overall ratings also gives a measure of the insight that the patient has into his or her own difficulties. We hypothesized that higher levels of reported executive dysfunction in AD patients, as well as insight (as measured by the discrepancy between informant and self-rated DEX scores) would be associated with higher perceived burden of care.

This single-site study was conducted at the Western Australian Centre of Health and Ageing of the University of Western Australia. Twentyfour participants with mild $\mathrm{AD}$ and their carers were recruited from metropolitan memory clinics and the community via advertising in the local media. The study was approved by the University of Western Australia and Royal Perth Hospital Ethics Committees, and participants and their carers gave written informed consent. Inclusion criteria included diagnosis of probable AD (NINCDS-ADRDA criteria), Mini-mental State Examination score $>17$, community living, and the availability of an informant. Clinical and cognitive assessment included the ADAS-Cog (global cognition), FAS word fluency task and the Digit-symbol coding task from the WAIS (executive and attention tasks), Cambridge Contextual Reading Task (an estimate of pre-morbid IQ), SF-36 questionnaire (measuring health and quality of life), and DEX questionnaire (subject version). Carers completed the Neuropsychiatric Inventory (NPI), DEX questionnaire (informant version), 
Zarit Burden Interview (ZBI), and the SF-36 questionnaire (rating their own health). Patients were also rated on the Clinical Dementia Rating (CDR).

The mean age of patients was 77.8 (SD 6.9) years and 14/24 patients were male. Sixteen of the carers were spouses and eight were adult children. Only six carers were male. We calculated the Pearson's correlation coefficient to examine the relationship between the scores in the ZBI and DEX rated by the carer $(r=0.44, p=0.034)$, the subject $(r=-0.24, p=0.259)$, and the difference between the two $(r=0.52, p=0.009)$. Other clinical and cognitive variables showed no obvious associations with ZBI scores.

The association between ZBI scores and the DEX was further evaluated by means of linear regression. The primary predictor variables of ZBI score (outcome variable) were as follows: in the first model, the DEX discrepancy score; in the second model, the DEX score according to the carer. Other variables were entered into the model one by one (forward stepwise) if their correlation with the ZBI was associated with a p-value of 0.2 or less. Variables that explained less than $5 \%$ of the model variance were removed. The first model was associated with $\mathrm{R}^{2}$ of $0.50(p=0.008)$, with the DEX discrepancy score contributing significantly to this model $(p=0.014)$. Other variables that contributed at least $5 \%$ of the variance were the CDR sum of boxes $(p=0.466)$, pre-morbid IQ $(p=0.186)$, and FAS score $(p=0.092)$. In the second regression model, $\mathrm{R}^{2}$ was $0.29(p=0.025)$, with the contribution of the DEX score according to the carer being significant $(p=0.036)$. The latter model also included pre-morbid IQ $(p=0.088)$.

The results of this study show that dysexecutive symptoms and reduced awareness of executive deficits are robust predictors of burden of care. Previous research has found that impaired awareness of memory deficits and BPSD are associated with increased burden scores (Donaldson et al., 1998; Rymer et al., 2002; Davis and Tremont, 2007). Our current findings suggest that dysexecutive dysfunction may be an even stronger predictor of carer burden. The mechanisms underlying such an association are unclear, but increasing conflict between the patient and his/her carer and loss of patients' "social graces" may all contribute to this unfavorable outcome for the carer.

Our results indicate that the DEX score of the carer, but not the patient, contribute to burden ratings. We did not, however, find any significant relationship between carer burden and the other measure of BPSD, the NPI. This suggests that, although the NPI and the DEX are both measuring behavioral disturbances, the DEX assesses behavioral problems (i.e. symptoms of executive dysfunction) that are particularly relevant or challenging to carers of patients with $\mathrm{AD}$. Therefore, the DEX (carer version and carer-patient discrepancy), or equivalent, seems to provide additional and clinically useful BPSD information to scales such as the NPI.

In conclusion, we found that carers' burden is associated with patients' decreased awareness of behavioral disturbances that result from executive dysfunction in mild $\mathrm{AD}$. If replicated, this finding would suggest that the systematic screening of executive dysfunction may contribute to the development 
of strategies designed to decrease the burden associated with the care of patients with AD.

\section{Acknowledgments}

This study was supported by an unrestricted project grant from the Australian Rotary Mental Health Fund. We thank all participants for their valuable contribution to this study.

\section{References}

Davis, J. D. and Tremont, G. (2007). Impact of frontal systems behavioral functioning in dementia on caregiver burden. Fournal of Neuropsychiatry and Clinical Neurosciences, 19, 43-49.

Donaldson, C., Tarrier, N. and Burns, A. (1998). Determinants of carer stress in Alzheimer's disease. International fournal of Geriatric Psychiatry, 13, 248-256.

Rymer, S., Salloway, S., Norton, L., Malloy, P., Correia, S. and Monast, D. (2002). Impaired awareness, behavior disturbance, and caregiver burden in Alzheimer's disease. Alzheimer Disease and Associated Disorders, 16, 248-253.

Wilson, B. A., Alderman, N., Burgess, P. W., Emslie, H. and Evans, J. J. (1996). Behavioural Assessment of the Dysexecutive Syndrome. Bury St Edmunds, U.K.: Thames Valley Testing Company.

Kathryn R. Bonney, Osvaldo P. Almeida, Leon Flicker, Kay Cox, Jonathan K. Foster, Frank M. van Bockxmeer and Nicola T. LAUTENSCHLAGER

WA Centre of Health \& Ageing, University of Western Australia, Perth, Australia Email:kathryn@cyllene.uwa.edu.au

doi:10.1017/\$1041610207006011

\section{"Alien face" in corticobasal degeneration syndrome: extending clinical features}

Corticobasal degeneration syndrome (CBDS) is a neurodegenerative disorder characterized by higher cortical dysfunctions associated with progressive asymmetric akinetic-rigid syndrome, and limb dystonia or focal myoclonus (Lang et al., 1994). One of the most typical and specific clinical features in CBDS is considered alien limb phenomenon, usually present in advanced disease stage (Doody and Jankovic, 1992).

Here we describe the case of a patient with CBDS complaining of a newly reported symptom, which, on account of its intriguing features, we termed "alien face" phenomenon. The patient gave her written consent to publication. 